\title{
Partage des données médicales du patient en utilisant le cloud computing
}

\section{Résumé}

La distribution automatique, électronique et sécurisée des données médicales du patient reste un défi auquel sont confrontés les systèmes de santé publics et privés
dans les pays dits en voie de développement. II n'en demeure pas moins dans les pays industrialisés. Ici les défis a relever sont différents.

Dans la plupart des systemes de santé public dans les pays en voie de développement, le dossier medical dans son etat actuel est basé sur du support papier et carnet actuel rend toute distribution de données médicales du patient difficitude. Le Utiliser les avancées technologiques de l'information et de la communication pourrait permettre de distribuer efficacement et de manière sécurisée les données médicales du patient.

Ce travail nous a permis d'une part d'analyser la situation actuelle du point de vue technique et technologique, et $d$ autre part d élaborer des solutions globales baseees sur les technologies de l'information et de la communication, permettant un meilleur partage des donnees. Le cloud computing est l'une des solutions technoloMots clés : Patient, données médicales, Para

nees médicales, cloud computing, carnet de santé

\section{Objectifs}

Proposer une approche de solution aux problèmes liés au partage des donnees médicales du patient. Parmi ces problèmes:

1- Lutilisation du support papier comme moyen d'enregistrement et de sauvegarde des donnees du palient

3- La difficulté d'accès aux données des patients

4- Archivage des données médicales des patients

\section{Méthode}

Numérisation et dématérialisation du carnet de santé

Création d'une base de donnée qui va centraliser les données des patients Concevoir un systeme dinformalion qui va permetre aux acteurs de la sante d'avoir un acces rapide et sécurise aux données

ên des données
Outils

Conception basée sur un architecture 3-tier

Single page Application

- Front End. NodeJS et Angular et lonic

Back End : Django

Base de données PostgreSq

Cons serveur web

Cloud : Openstack ou AWS
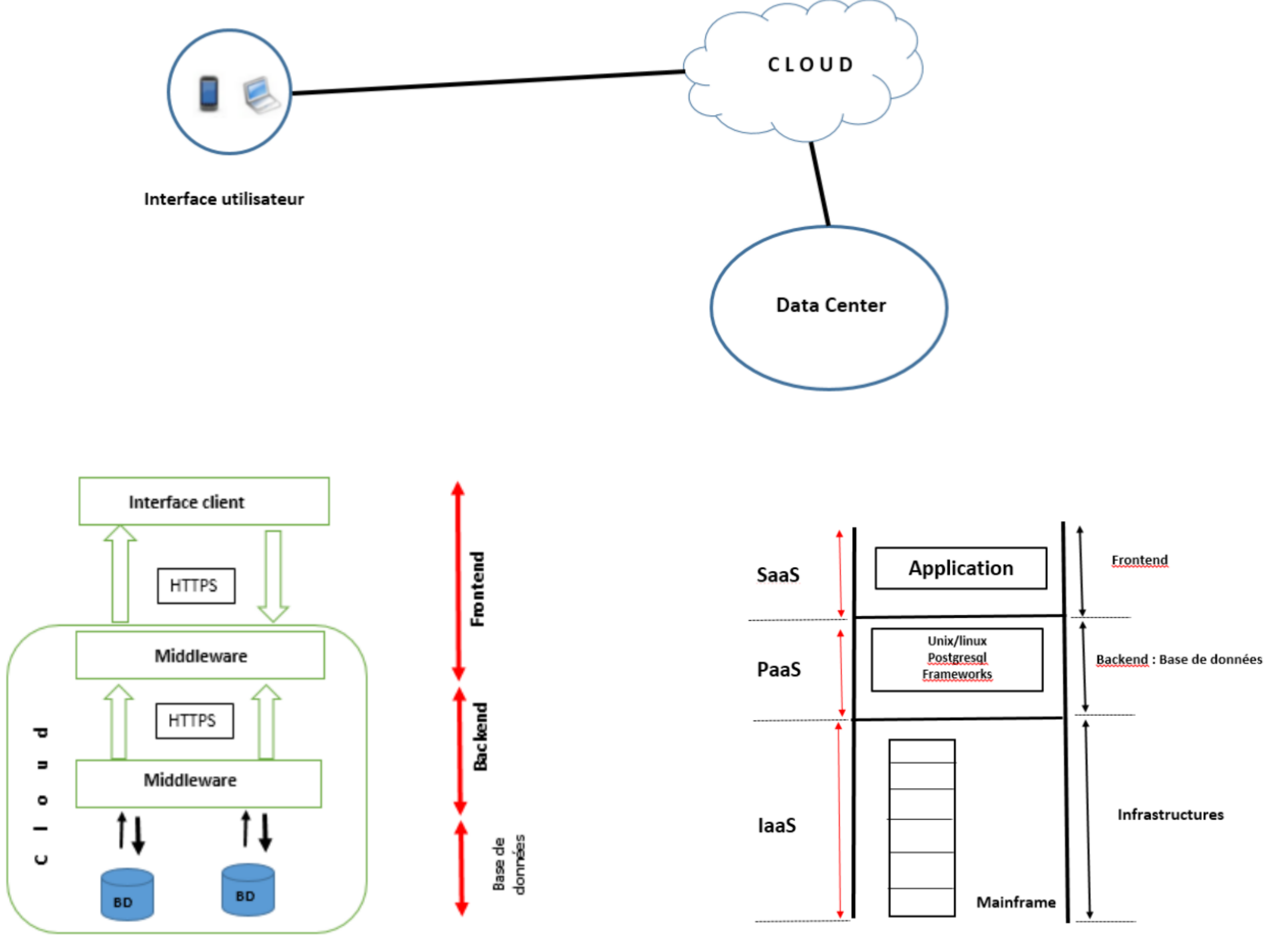

\section{Tests}
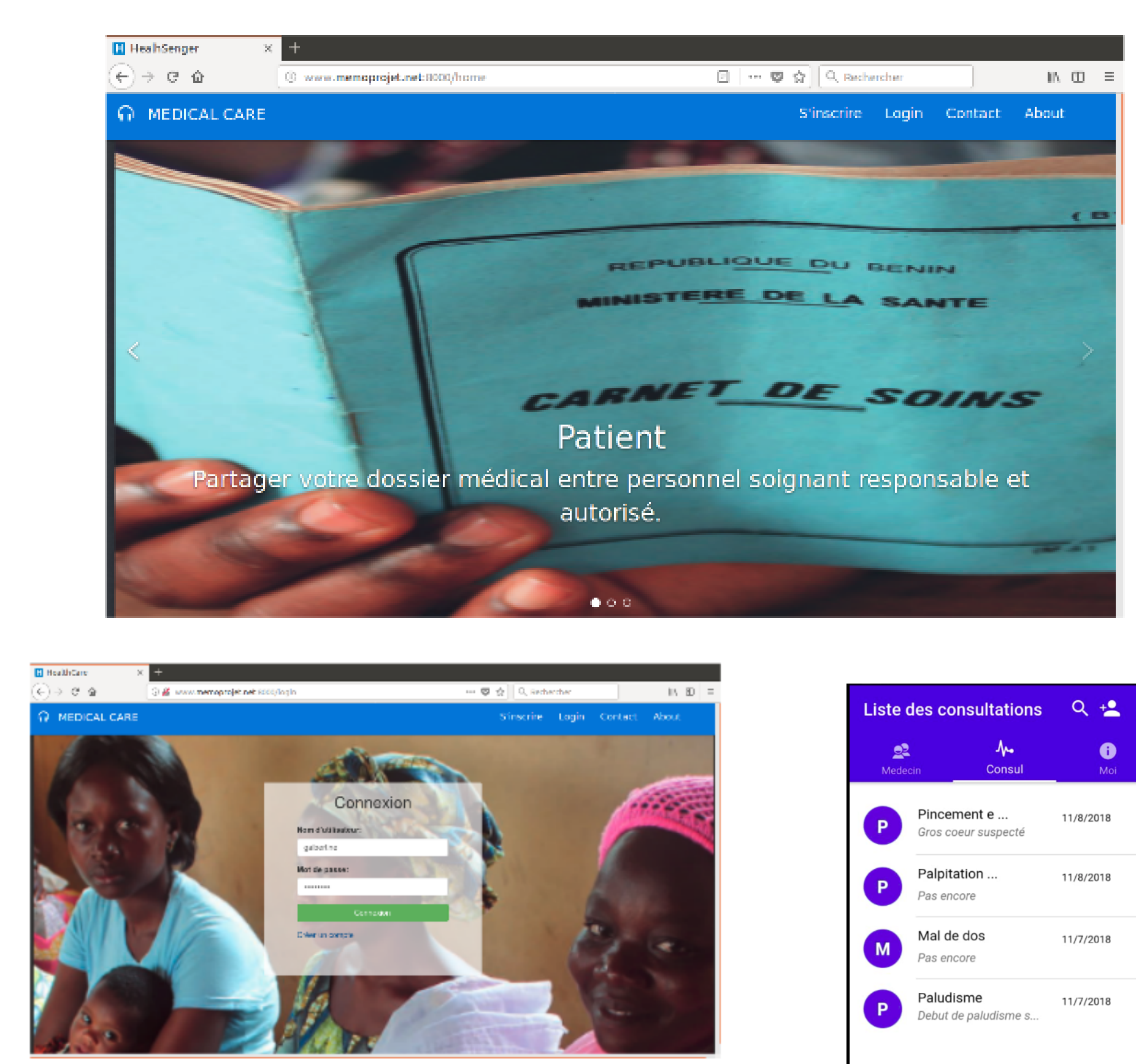

\section{Résultats}

\begin{tabular}{|c|c|c|c|}
\hline $\begin{array}{c}\text { Besoins } \\
\text { selon le } \\
\text { service du } \\
\text { cloud }\end{array}$ & Google & $\begin{array}{l}\text { Amazone Web Ser- } \\
\text { vices - EC2 }\end{array}$ & Microsoft Azur \\
\hline IaaS & $\begin{array}{l}\text { 8vCPU, RAM 26Go, } \\
\text { 32GoSSD coûte envi- } \\
\text { ron soit } 0.99 \$ / h \text { OS } \\
\text { Linux[22] }\end{array}$ & 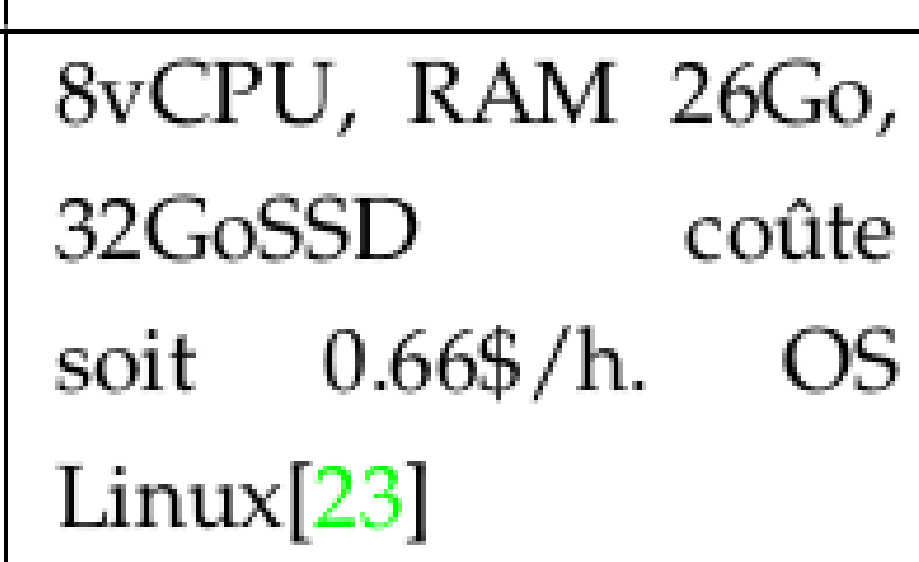 & $\begin{array}{l}\text { 8vCPU, RAM } 26 \mathrm{Go} \text {, } \\
56 \mathrm{DD} \text { temporaire } \\
\text { coûte } 0.559 \$ / \mathrm{h} \text { OS } \\
\text { Linux[24] }\end{array}$ \\
\hline PaaS & \multicolumn{3}{|c|}{$\begin{array}{l}\text { Installation de l'environnement de développement : Framewor } \\
\text { Django, NodeJs et PostgreSql pour la base de données }\end{array}$} \\
\hline Saas & & & \\
\hline
\end{tabular}

Cette solution, attendant une amélioration et sa phase de vulgarisaation sur lesquelles un travail est entrain d'être fait actuellement nous ne pouvons présenter ici des statistiques. Toutefois la disponibilité des
données des patients, l'accès, la numérisation et la dématérialisation sont des étapes importantes déjà franchises et vérifiables grâce aux differents précédemment effectués.

\section{Conclusion}

Dans les pays émergents, les données médicales des patients continuent de se faire encore sur du support papier avec les risques de perte , d'indisponibilité, de confidentialité d'intégrité nécessaire pour une meilleur prise en charge. Elle sont egalement archivees dans des conditions qui ne favorisent pas toujours une exploitation pour une prise en charge complète pour but de centraliser ces données afin de leur garantir un accès sécurisé et une conservation sur le long terme. 